\title{
Is Echocardiography Screening Necessary for All Patients on Admission to The Neonatal Intensive Care Unit?
}

\author{
Kenzo Sakurai* and Isamu Hokuto \\ Department of Pediatrics, St. Marianna University School of Medicine, Japan
}

*Corresponding author: Kenzo Sakurai, Department of Pediatrics, St. Marianna University School of Medicine, 2-16-1 Sugao, Miyamae-ku, Kawasaki, Kanagawa 216-8511, Japan

\section{ARTICLE INFO}

Received: 軕 January 02, 2020

Published: 㐩 January 10, 2020

Citation: Kenzo Sakurai, Isamu Hokuto. Is Echocardiography Screening Necessary for All Patients on Admission to The Neonatal Intensive Care Unit?. Biomed J Sci \& Tech Res 24(3)-2020. BJSTR. MS.ID.004066.

Keywords: Screening Echocardiography; Neonate; Congenital Heart Disease

Abbreviations: CHD: Congenital Heart Disease, NICU: Neonatal Intensive Care Unit, PH: Pulmonary Hypertension, TAPVC: Total Anomalous Pulmonary Venous Connection,

\section{ABSTRACT}

Background: Until now, cardiac structure was routinely assessed using echocardiography for all patients admitted to our neonatal intensive care unit (NICU). However, neonates who do not have cyanosis, cardiac murmur, distinct face traits, and external malformations usually have a normal cardiac structure. The purpose of this study was to evaluate the necessity of echocardiography screening on admission to our NICU.

Methods: A total of 82 patients with congenital heart disease (CHD) were admitted to our NICU between January 2013 and December 2017. We investigated their background and the trigger of the diagnosis by retrospectively analyzing medical records.

Results: The CHDs included 28 cases of ventricular septal defect (34\%), 12 of coarctation of the aorta (15\%), 12 of tetralogy of Fallot (15\%), nine of single ventricle including mitral valve atresia and tricuspid valve atresia (11\%), seven of double-outlet right ventricle (8\%), seven of atrioventricular septal defect (8\%), three of pulmonary atresia or critical pulmonary valve stenosis (4\%), two of great arteries transpositions $(2 \%)$, one of total anomalous pulmonary venous connection (TAPVC), and one of Ebstein's disease. Thirty-eight neonates (46\%) were diagnosed before birth. The number of neonates who presented with a specific face/external defect, cyanosis, and cardiac murmur were 33 (40\%), 36 (44\%), and 51 (62\%) respectively. No cases were diagnosed with CHD in the absence of cyanosis, cardiac murmur, specific face, and external malformation. The TAPVC case was diagnosed after a delay, despite presenting with cyanosis and respiratory disorder during admission.

Conclusion: Because neonates who did not present with cyanosis, cardiac murmur, specific face characteristics or external malformations did not have CHD, we conclude that echocardiography assessment is unnecessary for all patients. In addition, in cases of prolonged respiratory disorder, echocardiography assessment must be performed repeatedly to exclude TAPVC.

\section{Introduction}

Congenital Heart Disease (CHD) is an important cause of infant morbidity and mortality1). The reported prevalence of CHD at birth is 2 to 13 per 1000 live births 2,3 ). In the era of antenatal screening for CHD, infants presenting with an undiagnosed significant CHD are decreasing3). Echocardiography after birth is useful for the detection of CHD, but it requires some degree of examination technique and time. On the other hand, recent studies have recommended neonatal pulse oximetry to identify critical CHD4, 5). Pulse oximetry is a widely available, simple, safe, noninvasive, and accurate method to objectively quantify oxygen saturation, and thereby identify clinically undetectable hypoxia that occurs in most neonates with CHD5). To date, cardiac structure was routinely assessed using echocardiography for all patients admitted to our Neonatal Intensive Care Unit (NICU). However, neonates who do 
not have cyanosis, cardiac murmur, distinct facial traits, or external malformations usually have a normal cardiac structure. The purpose of this study was to evaluate the necessity of echocardiography screening on admission to our NICU.

\section{Methods}

A total of 82 patients with CHD were admitted to our NICU between January 2013 and December 2017. We investigated their background and the trigger for their diagnosis by retrospectively analyzing medical records. In all neonates, echocardiography was performed by a neonatologist using a Phillips IE 33 with a $12-\mathrm{MHz}$ transducer on admission. The gestational age of the subjects ranged from 35 weeks 1 day to 40 weeks 3 days, and their birthweight ranged from $2205 \mathrm{~g}$ to $3405 \mathrm{~g}$. Thirty-six neonates were male. Neonates with preterm patent ductus arteriosus were excluded in this study [1-7].

\section{Results}

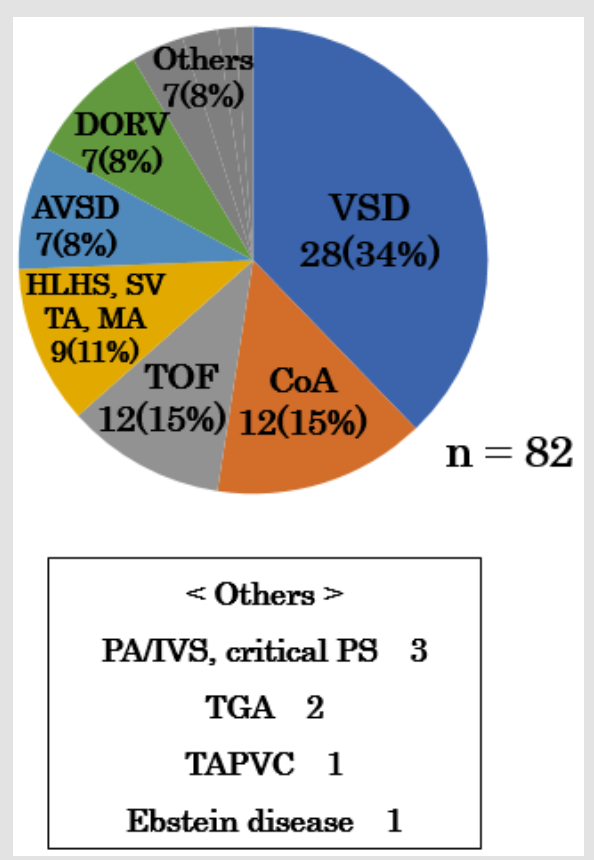

Figure 1: VSD; ventricular septal defect, CoA; coarctation of the aorta, TOF; tetralogy of Fallot, HLHS; hypoplastic left heart syndrome, SV; single ventricle, TA; tricuspid valve atresia, MA; mitral valve atresia, AVSD; atrioventricular septal defect, DORV; double outlet from right ventricle, PA/IVS; pulmonary atresia with intact ventricular septum, Critical PS; critical pulmonary stenosis, TGA; transposition of great artery, TAPVC; total anomalous of pulmonary venous connection.

The CHDs included 28 cases of ventricular septal defect (34\%); 12 of coarctation of the aorta (14\%); 12 of tetralogy of Fallot (15\%); nine of functional single ventricle (11\%) including mitral valve atresia, tricuspid valve atresia, hypoplastic left heart syndrome, and single ventricle; seven of double-outlet right ventricle (8\%); three of pulmonary atresia or critical pulmonary valve stenosis (4\%); two of great arteries transpositions (2\%); one of Total Anomalous Pulmonary Venous Connection (TAPVC); and one of Ebstein's disease (Figure 1). Thirty-eight neonates (46\%) were diagnosed before birth (Figure 2). CHDs were not diagnosed using fetal echocardiography included small VSD, simple CoA, TGA, and TAPVC. The number of neonates who presented with a specific facial features/external defect, cyanosis, or cardiac murmur on admission and that developed during admission were 33 (40\%), 36 (44\%), 19 (23\%), and 51 (62\%) respectively (Figures 3-6).

\section{Fetal diagnosis}

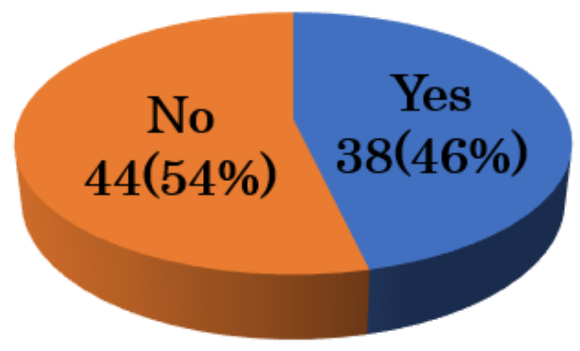

Figure 2: Thirty-eight neonates (46\%) were diagnosed before birth. The underlying pathology in cases who were not diagnosed using fetal echocardiography included small VSD, simple CoA, TGA, and TAPVC.

\section{Distinct face traits External malformations}

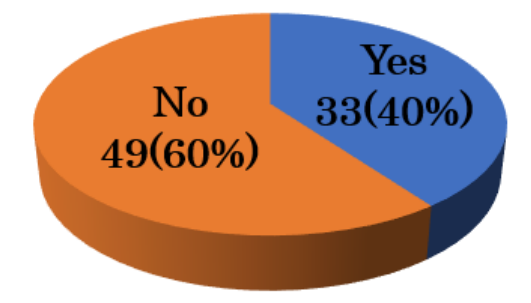

Figure 3: Thirty-three $(40 \%)$ neonates presented with specific facial features/external defects. The underlying pathology in patients who presented with specific facial features/external defects included trisomy 21, trisomy 18, trisomy 13, Noonan syndrome, and Goldenhar syndrome.

\section{Cardiac murmur on admission}

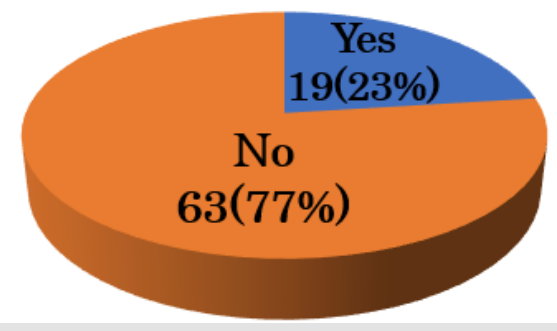

Figure 4: Nineteen neonates (23\%) presented with cardiac murmur on admission. The underlying pathology in patients with a cardiac murmur on admission included ventricular outflow tract stenosis (e.g., TOF, DORV, and critical PS). 


\section{Cardiac murmur during admission}

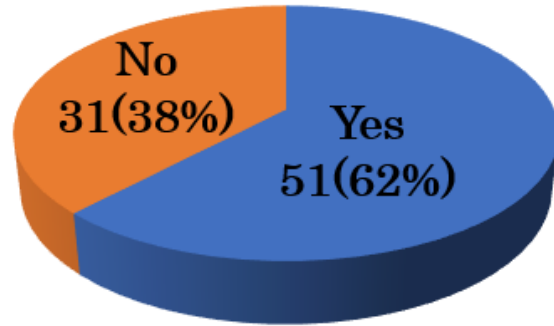

Figure 5: Fifty-one (62\%) neonates presented with cardiac murmur that developed during admission. The underlying pathology in patients who developed cardiac murmur during admission included VSD and AVSD, which gradually increases shunted blood flow after birth.

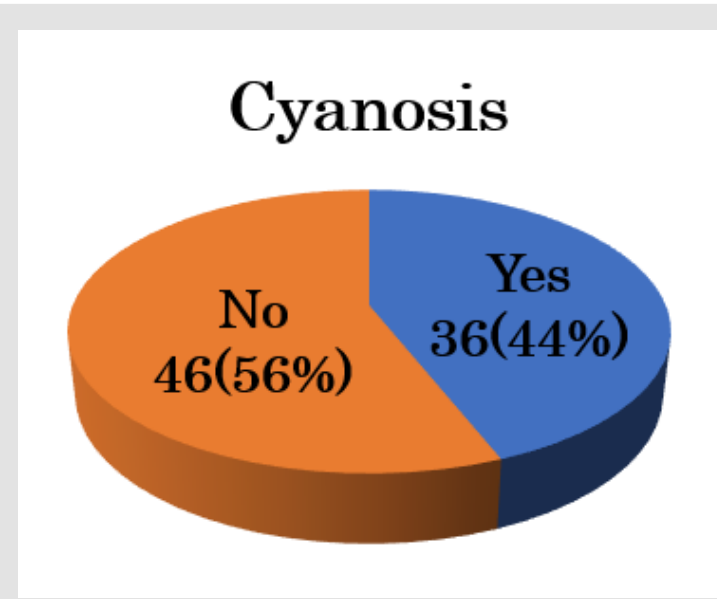

Figure 6: Thirty-six (44\%) neonates presented with cyanosis.

The underlying pathology in patients who presented with a specific facial features/external defects were trisomy 21, trisomy 18, trisomy 13, Noonan syndrome, and Goldenhar syndrome. The underlying pathology in patients who had cardiac murmur on admission included ventricular outflow tract stenosis (e.g., TOF, DORV, and critical PS). The underlying pathology in patients who developed a cardiac murmur during admission included VSD and AVSD, which gradually increases shunted blood flow after birth. No cases were diagnosed with CHD in the absence of cyanosis, cardiac murmur, specific facial features, or external malformation. The TAPVC case was diagnosed with a delay, despite presenting with cyanosis and respiratory disorder during admission.

\section{Discussion}

Neonatal echocardiography is performed for a wide variety of indications, including assessment of myocardial function and/or intravascular volume, diagnosis of Pulmonary Hypertension (PH), and detecting CHD. In recent years, neonatal echocardiography has been increasingly used to provide real-time data on the cardiovascular status of neonatal patients to identify hemodynamic compromise, aid in clinical decision making, and monitor treatment response6). However, neonatal echocardiography requires some degree of examination technique and time. In addition, neonates who do not have cyanosis, cardiac murmur, distinct facial features, or external malformations usually have a normal cardiac structure. In this study, no cases were diagnosed with CHD in the absence of cyanosis, cardiac murmur, specific facial features, or external malformation. Consequently, we recommend that echocardiography screening is not necessary for all patients on admission to the NICU. On the other hand, TAPVC was diagnosed after a delay, despite presenting with cyanosis and respiratory disorder during admission. In cases of prolonged respiratory disorder with cyanosis, echocardiography assessment must be performed repeatedly to exclude TAPVC and consult to pediatric cardiologist.

Recently, the usefulness of pulse-oximetry monitoring for detecting CHD has been reported7). A positive screen is defined as $\mathrm{SpO} 2<95 \%$ and/or differential cyanosis. Differential cyanosis is defined $>3 \%$ difference between the right hand and foot. In our hospital, we have routinely performed pulse-oximetry for neonates from 2017 to 2019. One neonate was diagnosed with CoA based on differential cyanosis. The limitations of this study are as follows. The number of subjects is low, and this is a retrospective study. This study did not consider X-ray findings on admission. The cardio-thoracic ratio and pulmonary vascular shadow help identify CHD. Therefore, further research is required regarding this aspect in Japan. Finally, because neonates who did not present with cyanosis, cardiac murmur, specific facial characteristics, or external malformations did not have CHD, we conclude that echocardiography assessment is unnecessary for all patients. In addition, in cases of prolonged respiratory disorder, echocardiography assessment must be performed repeatedly to exclude TAPVC.

\section{Disclosures}

\section{Conflict of Interest: None}

\section{Author Contribution}

All authors contributed to data collection and interpretation, and critically reviewed the manuscript. All authors approved the final version of the manuscript and agree to be accountable for all aspects of the work in ensuring that questions related to the accuracy or integrity of any part of the work are appropriately investigated and resolved.

\section{References}

1. Hill GD, Block JR, Tanem JB (2015) Disparities in the prenatal detection of critical congenital heart disease. Prenat Diag 35: 859-863.

2. Khoshnood B, Lelong N, Houyei L, Delezoide AL, Magny JF, et al. (2012) Prevalence, timing of diagnosis and mortality of newborns with congenital heart defects: a population-based study. Heart 98(22): 16671673.

3. Roberts T, Barton P, Auguste P (2012) Pulse oximetry as a screening test for congenital heart defects in newborn infants; a cost-effectiveness analysis. Arch Dis Child 97: 221-226. 
4. Bird T, Hobbs C, Cleves M, Canfield MA, Honein MA, et al. (2006) Nationa rate of birth defects among hospitalized newborns. Birth Defects Res A Clin Mol Teratol 76(11): 762-769.

5. Canfield M, Honein M, Yuskiv N (2006) National estimates and race/ ethnic-specific variation of selected birth defect in the United states, 1991-2001. Birth Defects Res A Clin Mol Terato 76(11): 747-756.

\section{ISSN: 2574-1241}

DOI: $10.26717 /$ BJSTR.2020.24.004066

Kenzo Sakurai. Biomed J Sci \& Tech Res

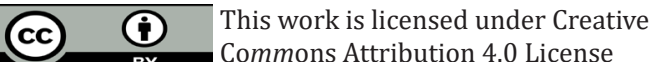

Submission Link: https://biomedres.us/submit-manuscript.php
6. Aisling S, Colm R, Adam T (2017) Incidental findings on routine targeted neonatal echocardiography performed in preterm neonates younger than 29 weeks' gestation. J Ultrasound Med pp. 0278-4297.

7. Alex R, William T, Gerard R, Carl Cooley, Praveen Kumar, et al. (2011) Strategies for implementing screening for critical congenital heart disease. Pediatrics 128(5): e1259.

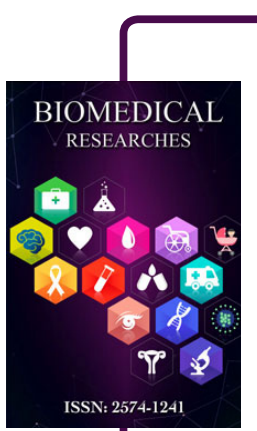

Assets of Publishing with us

- Global archiving of articles

- Immediate, unrestricted online access

- Rigorous Peer Review Process

- Authors Retain Copyrights

- Unique DOI for all articles

https://biomedres.us/ 